\title{
How does Brain Process Favorable Feedback with Low Possibility: An ERP Research
}

\author{
Fen Yu, Xiaoyong Xu, Jiannong Shi, \\ Institute of Psychology \\ Chinese Academy of Sciences, CAS \\ Beijing, China
}

\author{
Fen $\mathrm{Yu}$ \\ Graduate University \\ Chinese Academy of Sciences, CAS \\ Beijing, China
}

\begin{abstract}
Humans often learn from outcome feedback to modify the knowledge of objects/things, even to optimize subsequent strategies. Some studies have documented that participants divided outcomes into two sides in reward task: favorite and unfavorable outcome. But in real life we estimate outcome related closely at least two dimensions: whether the result will benefit us, and how frequent the result will occur. In this pilot ERP investigation, we adopt an elaborate gamble task to divide favorability evaluation and possibility evaluation, and assume FN and $\mathrm{P300}$ are indices for favorability and possibility respectively. Results show that FN were significantly more negative in the loss condition than in the gain condition, and P300 were significantly more positive in the gain/low-possibility condition than in the loss/high-possibility condition.
\end{abstract}

Keywords-favorability; possibility; ERP; P300; FN

\section{INTRODUCTION}

Humans often learn from feedback of environment to modify the knowledge of objects/things, even to optimize subsequent strategies. Almost immediately after feedback stimuli we evaluate those information automatically, and classify them in a basic category (e.g. good vs. bad, high vs. low possibility), especially when objects/things are relevant directly to ourselves. Previous researches have utilized event related potential (ERP) technology to invest outcome evaluation process in some interesting experiments. Some studies have documented that participants divided outcomes into two sides in reward task (i.e. gain vs. loss and neutral) and always preferred favorite to un-favorite outcome, which was detected by the significant difference on the amplitudes of some ERP components [1-3]. Whereas other researchers found that ERP components also showed frequency effect, that is to say people expected high frequent events rather than low frequent events, both in reward tasks [4] and in non-reward tasks.

Previous evidence identified two components of ERPs [feedback negativity (FN) and P300] engaged in feedback stimulus. FN is a negative deflection in the ERPs peaking approximately $250 \mathrm{~ms}$ following the onset of the negative outcome, which is distributed over the frontal scalp [1-2, 5]. A series of study showed that FN reflected the early appraisal of evaluation based on a binary classification of good versus bad outcome, e.g. accurate vs. inaccurate performance and monetary gain vs. bad [2-3, 5-7].
Another component that prior studies found was P300, a positive deflection with peak amplitude about $300 \mathrm{~ms}$ which is distributed over parietal scalp. P300 shows sensitivity to context updating [8-10], e.g. larger in oddball task following infrequent stimuli than frequent stimuli(note: "infrequent stimuli" in their experiment means the targets that appear really occasionally, while "low possibility stimuli" in our experiment focuses on the targets that participants expect appear occasionally.) [11]. Recently, the performance of P300 is also focused on after feedback which varies in participants' involvement in the tasks [12-13]. However, it remains a puzzle why negative emotion images elicit a larger P300 than do positive emotion images, whereas monetary gains elicit a larger P300 than do monetary loss.

According to the above, we found that those researches all cared that people would assess the feedback following just one kind of expectation, either hoped high frequent events or desired favorable rewards. Let's consider what will happen when we are confronted with results of a subsequent event in real life closely related with ourselves. We may estimate how much the result will benefit us and the probability of the benefit. For example, on lottery tickets buying, people realize the fact that a big bonus is a small probability event, but it does not prevent people from risk betting. Furthermore, knowledge of small probability of big bonus has a calming effect when people lose prize, or elicit a state of ecstasy when people win prize. So we assume that there are at least two dimensions of evaluation (favorability and possibility) in feedback processing, and they occur almost immediately and automatically. We query whether neural estimation of favorability of feedback stimuli is prior to that of possibility of feedback stimuli, or our brains sponsor the two categories simultaneously? Whether they have the similar amplitudes of neural arousals or different intensity? Even a third presumption: neural basis of favorability estimation involved more emotion related structures and less cognition related structures than did neural basis of possibility estimation. So we attempt to adopt ERP of high temporal resolution to investigate tentatively how brain process favorability and possibility estimation of feedback in a gambling task.

We assume FN and P300 are indices for favorability and possibility respectively. In this pilot investigation, we attempt to divide the feedbacks which participants receive into two 
levels of two types of categories. An elaborate gamble task is adopted.

\section{METHODS}

\section{A. Participants}

Ten undergraduates (6 females, aged between 19 and 22 years) from Beijing Normal University participated in the experiment. All the participants given written informed consent were right-handed and had no history of neurological impairment or psychiatric disorders. Participants were introduced to earn an extra bonus, except a basic pay of $¥ 20$ (about \$3), of about $¥ 5$ depended on the sum of their feedback scores during the task. Data from 1 participant were excluded due to excessive artifacts in latter part of EEG recording. So finally, only nine participants' data were analyzed. The study was approved by the local research ethics committee.

\section{B. Task and Procedures}

Participants were seated comfortably in front of a computer screen located at eye level at a distance of $75 \mathrm{~cm}$. Firstly a letter "I" was presented (randomly 100 200ms) to fix the participants' attention on the center of the screen. Then pairs, which participants pressed ' $F$ ' or " $J$ " as quickly as they could to choose one of the pair they took part in, were presented infinitely until acted. After an instant blank screen (randomly $400 \sim 600 \mathrm{~ms}$ ), participants saw the feedback of their choices (1000ms duration). All images were presented against a sliver gray background, and the inter-trial interval was $1,000 \mathrm{~ms}$. The experiment lasted approximately 20minutes, consisting of 2 blocks with180 trials each.

Before the formal test, participants were given detailed task introduction and a block of 20 trials for practice. Probability of feedback scores $(+7,-7,+8,-8)$ in the practice block depended on the response selected, which convinced participants that a priori probability of pairs is cue of following outcome score. However, in the formal experiment there were some sorts of tricks. Probabilities of the outcomes after pair land 2 were accordant with the pies chosen, while probabilities after pair 3 and 4 were delivered fifty to fifty chance, independent of predicted cues in the chosen pies. We set four total feedback scores of equal probability because previous researchers have found that the amplitude of the P300 was affected by a object probability of the stimuli [14-15]. After the test, a short interview included such questions as: "Do you think some pairs are easier to choose? If say "yes", which one?" "Please estimate your total scores and guess if you can get an extra bonus?"

It was a two by two (possibility (high vs. low); favorability (gain vs. loss)) within-subject design. In pair 3, positive outcome is defined as high-possibility gain, and negative outcome as low-possibility loss, for expectancy of two pies in pair 3 were both positive. Definitions in pair 4 were opposite, which positive outcome is defined as low-possibility gain, and negative outcome as high-possibility loss, for expectancy of two pies in pair 4 were both negative.

\section{ERP Recording}

The electroencephalogram (EEG) was recorded from 64 scalp sites using silver chloride electrodes mounted in a elastic cap (NeuroScan Inc. ) according to the International 10-20 system. Electrode impedances were kept below $5 \mathrm{k} \Omega$, The EEG and EOG were amplified with $0.05-100 \mathrm{~Hz}$ band-pass filter and sampled at $500 \mathrm{~Hz} / \mathrm{channel}$. The vertical and horizontal electrooculogram were recorded from electrodes placed above and below the left eye, and $1.5 \mathrm{~cm}$ lateral to the canthi of both eyes, respectively. All electrode recordings were referenced to the left mastoid. ERPs were recorded and analyzed with NeuroScan 4.3 software.

\section{ERP Analysis}

Ocular artifacts were corrected off-line with an eyemovement correction algorithm implemented in NeuroScan software. The data exceeded a threshold of $\pm 100 \mu \mathrm{V}$ were excluded from analysis, and filtered using a 1-30 Hz band-pass to remove low-frequency waves from the EEG. All trials referenced to the right mastoid. EEG epochs of $1200 \mathrm{~ms}$ (from $200 \mathrm{~ms}$ before the feedback stimulus onset to $1000 \mathrm{~ms}$ following feedback stimulus onset) were extracted for feedback-locked ERPs. These epochs were baseline corrected by subtracting the average activity of that channel from each sample. ERPs were created by group averaging EEG data by condition for each electrode and participant.

The FN amplitudes were measured as the peak amplitudes of the waveforms in a window of $220 \sim 300 \mathrm{~ms}$ after feedback, and the P300 amplitudes in a latter window of 320 400ms. Five midline electrodes( $\mathrm{Fz} 、 \mathrm{FCz} 、 \mathrm{Cz} 、 \mathrm{CPz} 、 \mathrm{Pz})$ of the $\mathrm{FN}$ and P300 were chosen into calculation for each participant. We chose these midline electrodes because former researchers had indicated that the FN/P300 effects were strongest on them. The Greenhouse-Geisser correction was applied to adjust $\mathrm{P}$ (significance).

\section{RESULTS}

\section{A. Behavior Results}

Paired sample T-test found that pair 1 did have a dominant choice $(\mathrm{t}(8)=9.735, \mathrm{p}<.001$, left in pair $1: 53.78 \pm 7.32$, right in pair 1: $6.22 \pm 7.32)$, the same to pair $2(\mathrm{t}(8)=14.272, \mathrm{p}<.001$, left in pair 2: $55.11 \pm 5.28$, right in pair 2: $4.89 \pm 5.28$ ). Pair 3 have no dominant choice( left in pair 3: $61.89 \pm 16.37$, right in pair 3: $58.11 \pm 16.37)$, while pair 4 have a dominant pattern $(\mathrm{t}(8)=-$ $4.384, \mathrm{p}<.01$, left in pair4 : $30.89 \pm 19.92$, right in pair 4: $89.11 \pm 19.92)$.

Means of reaction time didn't come into statistical analysis for two reasons: one was that experiment procedure didn't restrict reaction time; the other was that some extreme participants did absolute stable choice in pair with dominant choice, such as pair 1 or pair 2 .

\section{B. ERP Results}

Figure 2 illustrates the ERPs recorded on the five midline electrodes. ANOVA analysed the peak amplitudes and latency with factors of possibility (high-possibility vs. low), 
favorability (gain vs. loss), and electrode location ( Fz、FCz、 $\mathrm{Cz} 、 \mathrm{CPz} 、 \mathrm{Pz})$.

\section{FN (Feedback negativity)}

Each FN amplitudes reached maximum at $\mathrm{Fz}$ ( $\mathrm{Fz}(5.58 \pm 4.46), \quad \mathrm{FCz}(8.95 \pm 5.42), \quad \mathrm{Cz}(8.59 \pm 4.69)$, $\mathrm{CPz}(9.08 \pm 4.56), \mathrm{Pz}(8.43 \pm 4.43))$. ANOVA revealed a main effect of favorability $[\mathrm{F}(1,8)=9.096, \mathrm{p}<.05]$ and no difference of possibility. The amplitudes of FN were significantly more negative in the loss condition $(6.20 \pm 4.63 \mu$ $\mathrm{V})$ than in the gain condition $(10.06 \pm 4.28 \mu \mathrm{V})$. Further ANOVA with factors of possibility and favorability for each midline electrode failed to reach a main effect of possibility. An interaction of favor and location has been found $(\mathrm{F}(4,32)=5.321, \mathrm{p}<.05)$.

P300

Each P300 amplitude reached maximum at $\mathrm{CPz}$ (Fz(12.34.58 \pm 5.43$), \quad \mathrm{FCz}(16.21 \pm 6.35), \quad \mathrm{Cz}(14.86 \pm 5.61)$, $\mathrm{CPz}(15.71 \pm 5.37), \mathrm{Pz}(14.95 \pm 4.91))$. ANOVA revealed a main effect of favorability $[\mathrm{F}(1,8)=14.808, \mathrm{p}<.01]$ and no difference of possibility. The amplitudes of P300 were significantly more positive in the gain condition $(16.72 \pm 5.18 \mu$ $\mathrm{V})$ than in the loss condition $(12.91 \pm 5.49 \mu \mathrm{V})$. Further ANOVA with factors of possibility and favorability for each midline electrode revealed a main effect of possibility at $\mathrm{Pz}$ $[\mathrm{F}(1,8)=7.021, \mathrm{p}<.05]$, detailedly that the amplitudes elicited by low-possible feedback stimulus $(15.77 \pm 5.21 \mu \mathrm{V})$ were more positive than those of the high-possible feedbacks $(14.13 \pm 4.59$ $\mu \mathrm{V})$.

ANOVA of P300 latency revealed a main effect of favorability $(\mathrm{F}(1,8)=13.028, \quad \mathrm{p}<.01, \quad$ gain condition $(342.96 \pm 26.34)<$ loss condition $(369.82 \pm 13.63)$ ), and as well of possibility $(\mathrm{F}(1,8)=7.324, \mathrm{p}<.05$, high-possibility condition $(348.89 \pm 16.59) \quad<\quad$ low-possibility condition $(363.89 \pm 22.21))$.

\section{DISCUSSION}

Previous studies considered FN as reflection of the early appraisal of evaluation, which based on a binary classification of good versus bad outcome [2-3, 5-7]. Moreover Greg Hajack et al. $[4,16]$ insisted that the magnitude of FN was sensitive to violation of reward prediction. We replicated the former conclusion, but don't agree on the latter, for the previous tasks mainly investigated factors of reward value and magnitude, so they concluded that the best outcome equaled human prediction. But consider people's performance in real life, we hypnoses that people predict towards two dimensions, which are probability and favorability evaluation. We found that $\mathrm{FN}$ is only involved in favorability evaluation, not in possibility evaluation.

Previous studies have found that P300 was sensitive to object probability, such as in oddball task, higher P300 amplitude accompanied smaller probability stimuli [8-11]. And some other experiments showed that P300 amplitude was larger for unexpected feedback in gambling task [4], but in their tasks, the feedback stimuli probability is in accordance with participants' expectation of probability. We insist that P300 can be elicited by the feedback stimuli probability even if without prior probability expectation. In order to avoid the effect of feedback stimuli probability and strengthen the effect of subjective probability, we implied in this experiment that the percentage of color predicted the probability of the valence of subsequent outcome, whereas actual outcomes were presented half to half. We found that subjective probability effect is also occurred in P300 - higher P300 amplitude related with lower subject probability evaluation.

In our experiment, compared with the loss/high-possible conditions, amplitude of $\mathrm{P} 300$ is more positive in the gain/lowpossible condition. We prefer some researchers' explanation that P300 reflects participants' emotional involvement in the tasks[12-13] to others' explanation that P300 is sensitivity to context updating [8-10]. For higher arousal of some selffulfilment emotion and surprise beyond expectation is evoked by gain and low possibility respectively than loss and highpossibility, which motivates participants to keep interested in the tasks. Therefore we conclude that the performance of P300 reflects arousal of emotion, that higher arousal of emotion involved elicits more positive P300, especially when gains in low-possibility evoke highest potentials.

In this gambling task, just as our prior assumption, $\mathrm{FN}$ is index for favorability. However, out of our expectation, P300 is sensitive to both favorability and possibility. Illustrated in details, the amplitudes of FN were significantly more negative in the loss condition than in the gain condition, and the amplitudes of P300 were significantly more positive in the gain/high-possible condition than in the loss/ low-possible condition(note that the possibility effect in P300 was only located at $\mathrm{Pz}$ electrode). Significant differences between FN and P300 may suggest that favorability evaluation and possibility evaluation are two dimensions of feedback evaluation. We can deduce cautiously that our brain processes outcome evaluations involving favorability faster and deeper than others involving event possibility. But other differences of neural system respond to favorability and possibility is still not understood.

Even we had hoped to exclude the reward amplitude effect, we still found there were some pollutions. For example, we had hoped there were no dominant choices in pair3 (gain frame) and pair4 (loss frame) for similar expectancy, but behavior results showed that pair4 had dominant option ("8"). According to after-interview, the participants carefully balanced the expectancy and chose the slightly better one in pair4. While facing the pair 3 , they thought it was hopeful to gain, so they sometimes preferred to risk the larger value option, and neglected the better one. Therefore slight asymmetry effect of reward magnitudes in gain frame and loss frame would pollute the grand average ERPs. Moreover, in this experiment, two scales of subjective probability can't explain the relation trends between $\mathrm{P} 300$ and probability. All these answers depend on future studies.

\section{REFERENCES}

[1] C. B. Holroyd, G. Hajcak and J. T. Larsen, "The good, the bad and the neutral: electrophysiological responses to feedback stimuli," Brain Res, vol. 1105, pp. 93-101, Aug 112006. 
[2] G. Hajcak, J. S. Moser, C. B. Holroyd and R. F. Simons, "The feedbackrelated negativity reflects the binary evaluation of good versus bad outcomes," Biological Psychology, vol. 71, pp. 148-154, Feb 2006.

[3] C. B. Holroyd, J. T. Larsen and J. D. Cohen, "Context dependence of the event-related brain potential associated with reward and punishment," Psychophysiology, vol. 41, pp. 245-253, Mar 2004.

[4] G. Hajcak, C. B. Holroyd, J. S. Moser and R. F. Simons, "Brain potentials associated with expected and unexpected good and bad outcomes," Psychophysiology, vol. 42, pp. 161-170, Mar 2005.

[5] W. J. Gehring and A. R. Willoughby, "The medial frontal cortex and the rapid processing of monetary gains and losses," Science, vol. 295, pp. 2279-2282, Mar 222002.

[6] W. H. R. Miltner, C. H. Braun and M. G. H. Coles, "Event-related brain potentials following incorrect feedback in a time-estimation task: Evidence for a "generic" neural system for error detection," Journal of Cognitive Neuroscience, vol. 9, pp. 788-798, Nov 1997.

[7] C. B. Holroyd, M. G. H. Coles and S. Nieuwenhuis, "Medial prefrontal cortex and error potentials," Science, vol. 296, pp. 1610-1611, May 31 2002.

[8] E. Donchin and M. G. H. Coles, "Is the P300 Component a Manifestation of Context Updating," Behavioral and Brain Sciences, vol. 11, pp. 357-374, Sep 1988.

[9] S. Sutton, M. Braren, J. Zubin and E. R. John, "Evoked-Potential Correlates of Stimulus Uncertainty," Science, vol. 150, pp. 1187-\&, 1965.

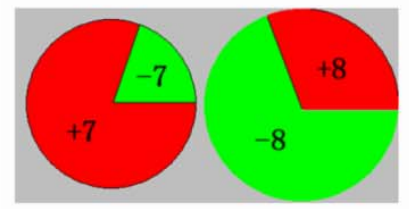

Pair 1

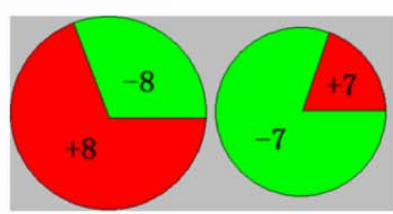

pair 2

[10] K. C. Squires, C. Wickens, N. K. Squires and E. Donchin, "Effect of Stimulus Sequence on Waveform of Cortical Event-Related Potential," Science, vol. 193, pp. 1142-1146, 1976.

[11] J. Policha and C. Margalab, "P300 and probability: comparison of oddball and single-stimulus paradigms," International Journal of Psychophysiology, vol. 25, pp. 169-176 1997.

[12] N. Yeung, C. B. Holroyd and J. D. Cohen, "ERP correlates of feedback and reward processing in the presence and absence of response choice," Cerebral Cortex, vol. 15, pp. 535-544, May 2005.

[13] N. Yeung and A. G. Sanfey, "Independent coding of reward magnitude and valence in the human brain," Journal of Neuroscience, vol. 24, pp. 6258-6264, Jul 142004.

[14] C. C. Duncan-Johnson and E. Donchin, "On quantifying surprise: the variation of event-related potentials with subject probability," Psychophysiology, vol. 14, pp. 456-467, 1977.

[15] T. S. Braver, D. M. Barch, J. R. Gray, D. L. Molfese, and A. Snyder, "Anterior Cingulate Cortex and Response Conflict: Effects of Frequency, Inhibition and Errors," Cereb. Cortex, vol. 11, pp. 825-836, September 1, 20012001.

[16] G. Hajcak, J. S. Moser, C. B. Holroyd and R. F. Simons, "It's worse than you thought: The feedback negativity and violations of reward prediction in gambling tasks," Psychophysiology, vol. 44, pp. 905-912, Nov 2007.

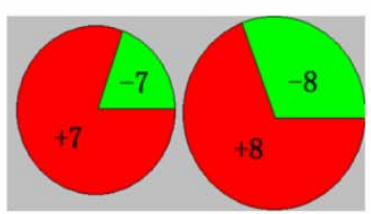

pair 3

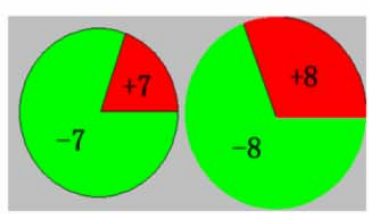

pair 4

Figure 1. Four types of pairs. In the pies, the portion of color predicted the probability of the value of subsequent outcome (red corresponded to gain while green to loss), and the radius indicated the absolute value subsequently (short corresponded to "7" while long to " 8 "). Pair 1 has a dominant option, for the expectancy of the left one is larger than that of the right one (left: $(+7) * 80 \%+(-7) * 20 \%=4.2$; right: $(-8) * 70 \%+(+8) * 30 \%=-3.2)$, the same to pair $2($ left: $(+8) * 70 \%+(-8) * 30=3.2$; right: $(-7) * 80 \%+(+7) * 20 \%=-4.2)$. Pair 3 and pair 4 have no dominant option, for the expectancy of the left one is close to that of the right one (left in pair 3 : $(+7) * 80 \%+(-7) * 20 \%=4.2$; right in pair $3:(+8) * 70 \%+(-8) * 30 \%=3.2$; left in pair $4:(-7) * 80 \%+(+7) * 20 \%=-4.2$; right in pair $4:(-8) * 70+(+8) * 30 \%=-3.2)$. Pies in each pair were counterbalanced. 


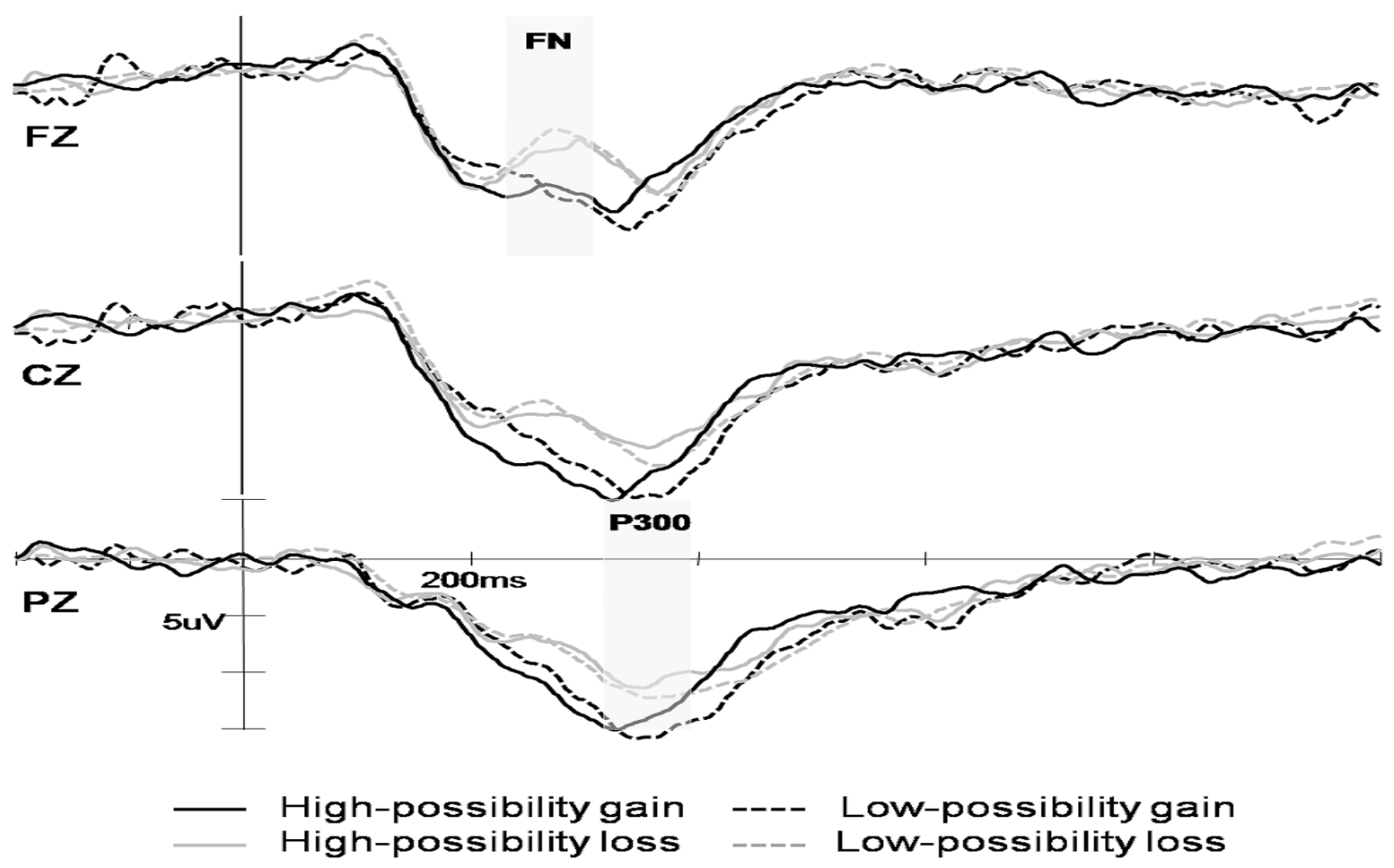

Figure 2. Grand average ERPs. 